including extraction of native stool, was assessed at a range of clinically relevant concentrations: Buhlmann EK-CAL 17\% (10 $\mu \mathrm{g} / \mathrm{g}), 12 \%$ (47 $\mu \mathrm{g} / \mathrm{g}), 19 \%$ (62 $\mu \mathrm{g} / \mathrm{g})$; PhiCal1 21\% (8 $\mu \mathrm{g} / \mathrm{g}), 24 \%$ $(10 \mu \mathrm{g} / \mathrm{g}), 18 \%(27 \mu \mathrm{g} / \mathrm{g})$; PhiCal2 19.5\% (18.9 $\mu \mathrm{g} / \mathrm{g})$. Inter-batch imprecision of ELISA analysis was lower: Buhlmann EK-CAL 8.6\% (30 $\mu \mathrm{g} / \mathrm{g})$, 5.8\% (129 $\mu \mathrm{g} / \mathrm{g})$; PhiCal1 6.2\% (39 $\mu \mathrm{g} / \mathrm{g}), 10.8 \%$ $(135 \mu \mathrm{g} / \mathrm{g})$; PhiCal2 $8.9 \%$ (33 $\mu \mathrm{g} / \mathrm{g})$. Functional sensitivity: Buhlmann EK-CAL $10 \mu \mathrm{g} / \mathrm{g}$; both PhiCal $20 \mu \mathrm{g} / \mathrm{g}$. Assays were found to be linear (without further sample dilution) up to $600 \mu \mathrm{g} / \mathrm{g}$ for EK-CAL, PhiCal1 $400 \mu \mathrm{g} / \mathrm{g}$, PhiCal2 $800 \mu \mathrm{g} / \mathrm{g}$. Mean recovery in spiked stool samples: Buhlmann EK-CAL 98\%, PhiCal1 83\%, PhiCal2 79\%.

Conclusion All three ELISA assays evaluated have relatively high coefficients of variation compared to other laboratory tests, due to heterogeneity of stool material and manual extraction/analysis. Results from different FC methods are not directly comparable, despite widespread adoption of single cut-offs. Using $50 \mu \mathrm{g} / \mathrm{g}$ cut-off, PhiCal1 performed better than Buhlmann EK-CAL in distinguishing IBD from IBS in our study. There is improved assay linearity using PhiCal2. Clinicians should be aware of type of ELISA methods employed when interpreting FC results, and cut-offs used should be fully evaluated.

Competing interests None declared.

\section{PM0-244 INFLIXIMAB REDUCES THE NEED FOR CORRECTION OF FISTULAE AND DRAINAGE OF ABSCESSES: A UK RETROSPECTIVE STUDY OF CROHN'S DISEASE PATIENTS}

doi:10.1136/gutjnl-2012-302514b.244

${ }^{1} \mathrm{C} D$ Wheeler, ${ }^{*}{ }^{1} \mathrm{R}$ Chipperfield, ${ }^{2} \mathrm{~T}$ Orchard, ${ }^{3} \mathrm{~J}$ Lindsay. ${ }^{1}$ Medical Affairs Department, Merck Sharp and Dohme, Hertfordshire, UK; ${ }^{2}$ Department of Gastroenterology, St Mary's Hospital, London, UK; ${ }^{3}$ Department of Gastroenterology, Royal London Hospital, London, UK

Introduction Crohn's disease affects 50-100 patients per 100000 in the population and typically follows a progressive course, with fistulae occurring in $17 \%$ to $43 \%$ of patients. The most common type of fistulae, perianal, have been shown to decrease quality of life and increase the likelihood of total colectomy. This retrospective study assessed incidence of procedures to correct fistulae and drain abscesses for a UK cohort of patients being treated with infliximab. Methods 18 UK centres participated in the study, including data from a total of 380 individuals with Crohn's disease who had received their first infusion of infliximab after 1 January 2003. Patients were eligible for inclusion with 12 months data prior to, and 24 months data post infliximab initiation, in their clinical record. Data on all investigations, clinic appointments, admissions and operations were extracted from the patient record in a standardised manner by members of the local clinical team. A prespecified statistical analysis plan compared healthcare resource utilisation at 12,18 and 24 months after the introduction of infliximab with resource utilisation during the 12 months prior to starting treatment.

Results In the 12 month period before initiation of infliximab therapy there were a total of 32 procedures within the study population to correct fistulae, treat severe anal fistulae or drain abdominal or peri-rectal abscesses. In the 24 -month period following initiation of infliximab therapy there were significantly fewer cumulative procedures (13 total). Procedures undertaken for correction of fistulae reduced from 12 (3.2\% of patients) in 12 months preinfliximab to 7 (1.9\% of patients) in the 24 months following infliximab initiation $(p<0.05)$. Treatment of severe anal fistulae was reduced from six cases $(1.6 \%)$ to $2(0.5 \%)$ ( $p<0.01)$. Procedures undertaken to drain either abdominal or peri-rectal abscesses reduced from $14(3.7 \%)$ to $4(1.1 \%)$ over the same period $(p<0.0001)$.

Conclusion In a large UK cohort of Crohn's disease patients, treatment with infliximab was shown to significantly reduce the need for surgical procedures relating to either fistulae correction or drainage of abscesses

Competing interests C Wheeler: Employee of: MSD, R Chipperfield Employee of: MSD, T Orchard: Grant/Research Support from: MSD, Warner Chilcott, Johnson and Johnson, Consultant for: Warner Chilcott, Ferring, Shire, Speaker bureau with: Warner Chilcott, Ferring, Shire, J Lindsay: Grant/Research Support from: MSD, Abbott, Shire, Consultant for: MSD, Abbott, Shire, GSK, Ferring, Warner Chilcott, Atlantic Healthcare, Speaker bureau with: MSD, Abbott, Shire, Ferring, Warner Chilcott.

\section{PMO-245 INFLIXIMAB REDUCES RATES OF EMERGENCY HOSPITAL ADMISSIONS AND SURGICAL PROCEDURES: A UK RETROSPECTIVE STUDY OF CROHN'S DISEASE PATIENTS}

doi:10.1136/gutjnl-2012-302514b.245

${ }^{1} \mathrm{C} D$ Wheeler, ${ }^{*}{ }^{1} \mathrm{R}$ Chipperfield, ${ }^{2} \mathrm{~T}$ Orchard, ${ }^{3} \mathrm{~J}$ Lindsay. ${ }^{1}$ Medical Affairs Department, Merck Sharp and Dohme, Hertfordshire, UK; ${ }^{2}$ Department of Gastroenterology, St Mary's Hospital, London, UK; ${ }^{3}$ Department of Gastroenterology, Royal London Hospital, London, UK

Introduction Crohn's disease affects 50-100 patients per 100000 in the population and typically follows a progressive course, with an estimated $33 \%$ of patients with active disease developing complications requiring hospitalisation or surgery in the first year of diagnosis. Up to $80 \%$ of patients require surgery at some point during the course of their disease. This retrospective study assessed the rates of emergency hospitalisation and surgery for a large UK cohort of patients before and after infliximab therapy.

Methods 18 UK centres participated in the study, including data from a total of 380 individuals with Crohn's disease who had received their first infusion of infliximab after 1 January 2003. Patients were eligible for inclusion with 12 months data prior to, and 24 months data post infliximab initiation, in their clinical record. Data on all investigations, clinic appointments, admissions and operations were extracted from the patient record in a standardised manner by members of the local clinical team. A prespecified statistical analysis plan compared healthcare resource utilisation at 12,18 and 24 months after the introduction of infliximab with resource utilisation during the 12 months prior to starting treatment.

Results In the 12-month period before initiation of infliximab therapy there were a total of 12 emergency admissions for surgical procedures. There were 176 unplanned emergency admissions in the same period to manage complications such as bowel obstruction or flare. In the 24-month period following initiation of infliximab therapy there were significantly fewer cumulative emergency admissions for surgical procedures ( 8 total, $\mathrm{p}=0.0184$ ) and management of complications (220 total, $p<0.0001$ ). The most common complications resulting in unplanned admissions included abscess, flare and intestinal obstruction which were reduced by half in the first year of infliximab therapy.

Conclusion In a large UK cohort of Crohn's disease patients, treatment with infliximab was shown to significantly reduce rates of emergency admissions for either surgical intervention or management of complications.

Competing interests C Wheeler: Employee of: MSD, R Chipperfield: Employee of: MSD, T Orchard: Grant/Research Support from: MSD, Warner Chilcott, Johnson and Johnson, Consultant for: Warner Chilcott, Ferring, Shire, Speaker bureau with: Warner Chilcott, Ferring, Shire, J Lindsay Grant/Research Support from: MSD, Abbott, Shire, Consultant for: MSD, Abbott, Shire, GSK, Ferring, Warner Chilcott, Atlantic Healthcare, Speaker bureau with: MSD, Abbott, Shire, Ferring, Warner Chilcott. 